\title{
Inovação em um contexto isomórfico nos Programas De Pós-Graduação Stricto Sensu em administração
}

Elida Furtado da Silva Andrade

Doutoranda do Programa de Pós-Graduação em Administração - PPGA/UNINOVE elidandrade@gmail.com

Josiane de Oliveira

Doutoranda do Programa de Pós-Graduação em Administração - PPGA/UNINOVE josiane_deoliveira@hotmail.com

Emerson Antonio Maccari

Doutor em Administração pelo Programa de Pós-Graduação em Administração - PPGA/FEA USP. Professor do Programa de Pós-Graduação em Administração da UNINOVE emersonmaccari@gmail.com

Heloisa Hollnagel

Doutora em Ciências pela USP. Profa. do Mestrado em Gestão Pública da UNIFESP hollnagelhelo@gmail.com

Editor Científico: José Edson Lara

Organização Comitê Científico

Double Blind Review pelo SEER/OJS

Recebido em 06.05.2018

Aprovado em 20.06.2018

Este trabalho foi licenciado com uma Licença Creative Commons - Atribuição - Não Comercial 3.0 Brasil 


\title{
Resumo
}

O sistema de avaliação da pós-graduação, implantado pela CAPES, propicia identificar ações que possam levar os programas a atingirem padrões internacionais de qualidade e fornece informações para subsidiar decisões relativas a autorizações, credenciamentos e distribuição dos recursos (fomento à pesquisa), de forma que estes possam atingir os resultados desejados. Entretanto, como todos os programas, independente da área de avaliação, devem seguir os mesmos quesitos e métricas do sistema de avaliação, esses programas, podem tender ao isomorfismo coercitivo. Neste trabalho procurou-se verificar como se dá a inovação nos Programas de Pósgraduação em Administração stricto sensu em um ambiente isomórfico. O método utilizado foi a pesquisa documental, realizada em programas de excelência da área de conhecimento Administração. Como resultado do estudo observou-se que, pressionados pelos mesmos requisitos e em busca da excelência no processo avaliativo, os programas passam por processo de isomorfismo coercitivo e que poucos são os programas que promovem ações diferenciadas, as quais ocorrem, principalmente, no que tange à inserção social e à internacionalização. Como exemplo dessas ações, citam-se o programa de bolsas taxa e o módulo internacional obrigatório na matriz curricular, instituídos concomitantemente por somente um dos programas de pós-graduação, dentre os pesquisados.

Palavras-chave: Isomorfismo; inovação; pós-graduação; avaliação

\section{Innovation in an isomorphic context in the Stricto Sensu Postgraduate programs in administration}

\begin{abstract}
The graduate evaluation system, implemented by CAPES, allows identifying actions that can lead the programs to reach international quality standards and provides information to guide decisions regarding authorizations, accreditations and distribution of resources (research promotion), in a manner that they can achieve the desired results. However, since all programs, regardless of the evaluation area, must follow the same requirements and metrics of the evaluation system, these programs may tend to coercive isomorphism. In this work, we tried to verify how the innovation in the Graduate Programs in Administration stricto sensu happens in an isomorphic environment. The method used was documental research, carried out in programs of excellence of the administration knowledge area. As a result of the study, it was observed that, pressed by the same requirements and in search of excellence in the evaluation process, the programs undergo a process of coercive isomorphism and that there are few programs that promote differentiated actions, which occur mainly, social insertion and internationalization. As an example of these actions, mention is made of the fee scholarship program and the compulsory international module in the curricular matrix, which were concurrently instituted by only one of the postgraduate programs among those were surveyed.
\end{abstract}


Keywords: Isomorphism, innovation, postgraduate, evaluation

\section{Innovación en un contexto isomórfico en los programas de Post-Graduación Stricto Sensu en administración}

\section{Resumen}

El sistema de evaluación del posgrado, implantado por la CAPES, propicia identificar acciones que puedan llevar a los programas a alcanzar estándares internacionales de calidad y proporciona informaciones para subsidiar decisiones relativas a autorizaciones, acreditaciones y distribución de los recursos (fomento a la investigación), de forma que puedan alcanzar los resultados deseados. Sin embargo, como todos los programas, independientemente del área de evaluación, deben seguir los mismos requisitos y métricas del sistema de evaluación, esos programas, pueden tender al isomorfismo coercitivo. En este trabajo se buscó verificar cómo se da la innovación en los Programas de Postgrado en Administración stricto sensu en un ambiente isomórfico. El método utilizado fue la investigación documental, realizada en programas de excelencia del área de conocimiento Administración. Como resultado del estudio se observó que, presionados por los mismos requisitos y en busca de la excelencia en el proceso de evaluación, los programas pasan por proceso de isomorfismo coercitivo y que pocos son los programas que promueven acciones diferenciadas, las cuales ocurren, principalmente, en lo que se refiere a la inserción social ya la internacionalización. Como ejemplo de estas acciones, se citan el programa de becas y el módulo internacional obligatorio en la matriz curricular, instituidos concomitantemente por solamente uno de los programas de postgrado, entre los encuestados.

Palabras clave: Isomorfismo, innovación, postgrado, evaluación

\section{Introdução}

A educação continuada, subsequente à graduação, está consolidada como uma estratégia de aprimoramento da formação profissional necessária para ter sucesso no mercado de trabalho. Iniciada nos anos de 1930, a pós-graduação no Brasil está em pleno desenvolvimento. O primeiro modelo proposto para a pós-graduação brasileira foi moldado no padrão europeu e implementado, de maneira informal, no curso de Direito da Universidade do Rio de Janeiro, na Faculdade Nacional de Filosofia e na Universidade de São Paulo (Santos, 2003).

Em 1965, o Conselho Federal de Educação publicou o parecer 977, relatado pelo professor Newton Sucupira, cujo objetivo instava a definição e a regulamentação dos cursos de pós-graduação. Neste parecer, o professor adotou o modelo de pósgraduação norte americano para estabelecer o novo padrão para a pós-graduação brasileira (Sucupira, 1980). 
Com o intuito de aferir o nível de qualidade dos cursos ofertados, a Capes iniciou, em 1976, o processo de avaliação da pós-graduação, a fim de "assegurar a existência de pessoal especializado em quantidade e qualidade suficientes para atender às necessidades dos empreendimentos públicos e privados que visam ao desenvolvimento do país" (CAPES, 2017a).

Neste sentido, Martins, Maccari, Storopoli e Andrade (2013) afirmam que há uma preocupação das Instituições de Ensino Superior (IES) no aprimoramento e monitoramento do desempenho dos seus programas de pós-graduação por meio de sistemas de auto avaliação (interno) e desempenho de seus programas no sistema de avaliação da CAPES (externo). Este último, de acordo com Belloni (2000), em uma visão macro, busca identificar ações que levam os programas de pós-graduação a atingirem padrões internacionais e, em uma visão micro, contribui para que o poder público consiga tomar decisões relativas a autorizações e credenciamentos dos cursos, bem como na distribuição dos recursos, para que os programas possam atingir os resultados desejados. Cabe destacar que existem critérios gerais de avaliação pela CAPES: produção científica dos docentes e discentes, a formação do corpo docente, a qualidade da formação dos alunos, a formação de redes de pesquisa bem como o impacto social do programa.

A partir de 2008, foi inserida no Sistema de Avaliação da Capes, no quesito "Proposta do Programa", a exigência da descrição de inovações nas práticas de ensino. Em função disso, nos anos subsequentes, os Programas começaram a relatar e aprimorar suas experiências em relação às inovações nas práticas de ensino, com vistas a atender a essa nova exigência do sistema de avaliação. Entretanto, como todos os programas devem seguir os mesmos quesitos e métricas do sistema de avaliação da CAPES, estes podem tender ao isomorfismo coercitivo, identificado por DiMaggio \& Powell (1983), quando afirmam que as regulamentações governamentais e as expectativas culturais são capazes de impor uniformidades às organizações (Martins et al., 2013). Diante do exposto, este estudo tem o objetivo verificar como se dá a inovação nos Programas de Pós-graduação em Administração stricto sensu em um ambiente isomórfico.

Este estudo é apresentado em cinco seções, incluindo esta introdução. $\mathrm{Na}$ segunda seção apresentam-se as bases teóricas que darão suporte para a análise 
dos resultados, na terceira seção o método de pesquisa é descrito, na quarta seção são apresentados os resultados e a discussão e posteriormente, as considerações finais.

\section{Teoria Institucional}

A escola institucional, em discussões dos ambientes institucionais e seus efeitos sobre as estruturas e processos organizacionais, é considerada um suporte vital na pesquisa corrente sobre a adaptação organizacional (Hirsch, 1972; McNeil \& Minihan, 1977; Zald, 1978; Dowling \& Pfeffer, 1975; Hirsch, 1975; Meyer \& Rowan, 1977).

Guarido Filho e Machado-da-Silva (2001), afirmam que a noção de contexto institucional das organizações é resultado de uma concepção mais estruturada e extensiva do ambiente, englobando, além da dimensão econômica, a sociocultural, fazendo com que os conceitos advindos da teoria institucional relativos ao ambiente técnico e ao ambiente institucional deixem clara essa análise mais aprofundada. Desta forma, a atividade organizacional sofre influência tanto dos aspectos econômicos e regras institucionalizadas, quanto de um amplo sistema de crenças e valores ambientais (Meyer \& Rowan, 1977). Diante disso, pode-se afirmar que as organizações submetidas a pressões do ambiente técnico e do ambiente institucional são avaliadas, respectivamente, pela eficiência e pela adequação às exigências sociais (Machado-da-Silva, Fonseca, \& Fernandes, 1999).

Para DiMaggio \& Powell (1983), as organizações, na fase inicial do seu ciclo de vida, possuem grandes diferenças umas das outras no que tange à abordagem e à forma, porém, a partir de determinado momento, quando encontram-se mais consolidadas, passam a tornar-se cada vez mais homogêneas e a melhor definição para explicar esse processo é a de "isomorfismo" que, na definição de Hawley (1968), pode ser entendido como um processo que limita e induz uma unidade em determinada população a tornar-se semelhante a outras pelo fato delas estarem inseridas em um mesmo contexto.

Com base nesta perspectiva, as organizações, além de competirem pela disponibilidade de recursos e mercado consumidor, competem também pela legitimidade contextual de suas ações e estratégias. Ademais, consideram que forças vinculadas ao sistema de valores ambientais afetam o direcionamento estratégico da 
organização, bem como influenciam a dimensão estrutural da aprendizagem organizacional. Esta abordagem sugere que à medida que há um aumento na compatibilidade com as características ambientais, ocorrem mudanças nas características das organizações, e que a quantidade de organizações em determinada população é em função da capacidade de sustentação do ambiente e a diversidade de configurações organizacionais é isomórfica à diversidade ambiental (DiMaggio \& Powell, 1983).

Apoiados em Meyer (1979) e Fennell (1980), DiMaggio e Powell (1983) sustentam que existem dois tipos de isomorfismo: Isomorfismo Competitivo e o Isomorfismo Institucional. O primeiro pressupõe uma racionalidade sistêmica com foco na competição no mercado, na mudança de nichos e em medidas de adequação. $O$ segundo, diz respeito ao fato das organizações terem uma tendência a utilizar linhas de ação já definidas na sociedade, garantindo sua sobrevivência e legitimidade, independentemente de sua eficiência, orientando-se, pelo contexto, pelas regras e significados já institucionalizados nas estruturas, o que torna suas práticas cada vez mais homogêneas dentro de um ambiente.

Foram identificados três mecanismos pelos quais as mudanças isomórficas institucionais podem ocorrer:

i) isomorfismo coercitivo resulta da interação entre pressões formais e informais exercidas sobre as organizações por outras organizações das quais elas dependem e das expectativas culturais da sociedade em que estas atuam. Tais pressões podem ser sentidas como coerção, persuasão ou convite para se unirem em conluio (DiMaggio \& Powell, 1983). Segundo Meyer e Rowan (1977) à proporção que Estados e grandes organizações expandem seus domínios, cada vez mais regras institucionalizadas e legitimadas vão surgindo e as consequências para essas organizações são tornarem-se gradativamente mais homogêneas dentro de determinados domínios e em torno de rituais das instituições de maior porte.

ii) isomorfismo mimético ocorre quando as tecnologias organizacionais não são plenamente compreendidas e, em face da incerteza decorrente de problemas tecnológicos, objetivos conflitantes e exigências ambientais, algumas organizações podem tomar outras como modelo, ou seja, uma organização imita os arranjos 
estruturais e procedimentos bem sucedidos implementados por outra organização (March \& Olsen, 1976; DiMaggio \& Powell, 1983).

iii) isomorfismo normativo está associado à profissionalização, ou seja, atores externos podem induzir uma organização a se adequar a seus pares, determinando que ela desenvolva uma tarefa particular e especificando a classe profissional responsável pelo seu desempenho.

A pressão institucional exercida pelo Sistema de Avaliação da CAPES sobre os programas de pós-graduação pode ser classificada como isomorfismo coercitivo, pois visa à homogeneização a fim de que se enquadrem no padrão de qualidade estabelecido como referencial, para que seja possível a aferição e atribuição dos conceitos. Esses conceitos são utilizados com o intuito de legitimação, de modo que possam obter apoio, como bolsas e auxílio fomento, além de reconhecimento para validade nacional dos diplomas. Além disso, as pressões decorrentes desse Sistema de Avaliação afetam a forma como os programas de pós-graduação administram as suas atividades, a maneira como o corpo docente interpreta a avaliação e interage com o corpo discente (Machado-da-Silva, 2003; InfoCapes, 2000).

Nesta linha, Machado-da-Silva (2003) defende que os docentes dos programas de pós-graduação stricto sensu, ao se encontrarem diante de pressões institucionais de natureza coercitiva, têm a opção de agir de forma estratégica e diversa e mesmo assim atender às exigências, com base em seus valores e crenças.

Um dos caminhos seguidos para esse intento é por meio da inovação, cujo tema será objeto de estudo do próximo tópico.

\subsection{Inovação}

O primeiro vocábulo que deu origem ao termo inovação foi identificado no século $X V$, o qual é oriundo dos vocábulos ennouaço e emnouaçam, que se modificaram através dos tempos, até formar o termo inovação (Cunha \& Sobrinho, 2007).

Os trabalhos de Shumpeter, da década de sessenta, são reconhecidos na literatura como a origem dos estudos sobre inovação. $O$ autor amparou sua proposta de teoria do desenvolvimento econômico na premissa de que existe um equilíbrio entre os sistemas de oferta e procura e que cabe ao empreendedor, através da 
inovação, romper com este equilíbrio. Segundo esta teoria, a inovação seria o principal impulsionador da economia (Boava, Macedo, Magalhães, \& Silva, 2013).

Schumpeter (1982) conceitua inovação como tudo aquilo que não é familiar ao consumidor, ou seja, a introdução de um bem que é novidade para os consumidores. Outro autor, Christensen (2003), distingue as inovações em dois tipos: as disruptivas e as incrementais. A incorporação de novos elementos no produto/serviço, sem que haja uma alteração das suas funções básicas, é conceituada como inovação incremental (Bessant, Tidd, \& Pavitt, 2008).

Por inovação disruptiva (ou descontinuada) pode-se entender aquelas que rompem e redefinem os modelos em vigor, podendo refletir em novos lançamentos de produtos/serviços, por vezes mais simples, econômicos e convenientes, a fim de satisfazer uma dada necessidade ou, até mesmo, criar uma necessidade que, até o momento, era inexistente. O entendimento contemporâneo da inovação como fenômeno atrelado à geração de ganho qualitativo, independentemente de perspectiva setorial, se dissemina entre vários autores (Miles, 2005; Nelson \& Winter, 2005; Gualberto \& Moreira, 2013) e está presente em ambientes diversos, tais como na educação, na tecnologia, na economia, dentre outros (Palma \& Forster, 2011).

No Brasil, o Decreto ㄲo 5.563/2005 dispõe sobre incentivos à inovação e à pesquisa científica e tecnológica no ambiente produtivo, entendendo a inovação como a "introdução de novidade ou aperfeiçoamento no ambiente produtivo ou social que resulta em novos produtos, processos ou serviços" (Brasil, 2005), conceito próximo ao de Schumpeter. No campo da Educação, em especial na educação superior, destaca-se o grande desafio da pós-graduação na formação não só de pesquisadores, mas de futuros professores, imbuídos de um conhecimento que abranja, além da racionalidade humana, a subjetividade, a criatividade e a inovação (Barreto \& Martínez, 2007).

Para Martínez (1997), a criatividade é considerada ao mesmo tempo algo "novo e valioso" em um campo específico da ação humana, enquanto a inovação é uma "sequência de atividades pelas quais um novo elemento é introduzido em uma unidade social, com a intenção de beneficiar" (Bruno-Faria, 2003). Assim, o ensino em nível de pós-graduação é "convidado" a inovar, buscando novas estratégias, organizações de ideias, formulações de conceitos e percepções. Também a 
orientação acadêmica deve seguir este movimento, que se apresenta como desafio e conquista (Barreto \& Martínez, 2007).

É senso comum que a relação entre internacionalização de empresas e inovação é diretamente proporcional, sendo considerada uma vantagem competitiva para a organização, não só pelo acesso a novas tecnologias (e possibilidades de inovação), mas por adquirirem novos conhecimentos e visões culturais distintas (Ribeiro, 2016). Esses aspectos são muito importantes em Programas de formação de recursos humanos como a Pós-graduação.

\subsection{Evolução da pós-graduação no Brasil}

O termo "pós-graduação" foi utilizado pela primeira vez na década de 1940, no Artigo 71 do Estatuto da Universidade do Brasil. No entanto, foi com a proposta de implantação de uma pós-graduação nos moldes europeus no Estatuto das Universidades Brasileiras que a pós-graduação teve início no Brasil, na década de 1930 (Santos, 2003). Esse modelo foi implementado no curso de Direito da Universidade do Rio de Janeiro, na Faculdade Nacional de Filosofia, e na Universidade de São Paulo. Naquela época, conforme explica Balbachevsky (2005), chegaram às universidades brasileiras um significativo número de professores estrangeiros que trouxeram o primeiro modelo institucional para os estudos na pósgraduação no Brasil, baseado na relação tutorial entre professor e aluno.

Em 15 de janeiro de 1951, o Presidente Dutra sancionou a Lei № 1.310 que criava o Conselho Nacional de Pesquisas/CNPq, como autarquia vinculada à Presidência da República. Essa lei foi chamada pelo Almirante Álvaro Alberto da Motta e Silva, autor da proposta de criação do Conselho, de "Lei Áurea da pesquisa no Brasil" (CNPQ, 2017).

O Conselho tinha como finalidades promover e estimular o desenvolvimento da investigação científica e tecnológica, mediante a concessão de recursos para pesquisa, formação de pesquisadores e técnicos, cooperação com as universidades brasileiras e intercâmbio com instituições estrangeiras (CNPQ, 2017). Neste sentido, durante a década de 1950 foram firmados acordos entre os Estados Unidos e o Brasil que deram origem a convênios entre escolas e universidades norte-americanas e 
brasileiras por meio do intercâmbio de estudantes, pesquisadores e professores (Santos, 2003).

Com o objetivo de "assegurar a existência de pessoal especializado em quantidade e qualidade suficientes para atender às necessidades dos empreendimentos públicos e privados que visam ao desenvolvimento do país", foi criada pelo Decreto oㅜ 29.741, em 11 de julho de 1951, a Campanha Nacional de Aperfeiçoamento de Pessoal de Nível Superior, atual Capes (CAPES, 2017a).

Entretanto, o grande impulso para os cursos de pós-graduação no Brasil, conforme Moritz, Pereira, Moritz, e Maccari (2013), só aconteceu na década de 1960, com a publicação do Parecer 977 do Conselho Federal de Educação, que regulamentou os cursos de pós-graduação no Brasil, criando os níveis de mestrado e doutorado, com muita semelhança à estrutura americana. Neste parecer, a pósgraduação foi defendida pelo professor Newton Sucupira como uma necessidade imperativa por razões que afetavam diretamente, não apenas o desenvolvimento da ciência no Brasil, mas o pleno desempenho da própria graduação (Bomeny, 2001).

Nesse novo contexto, considerando as especificidades do ensino superior brasileiro, surgiram discussões acerca da necessidade de investimentos em pesquisa e ciência para o desenvolvimento do país entre os meios político e acadêmico (Paixão, Barbosa, \& Neves, 2009). Assim, em 1976, a Coordenação de Aperfeiçoamento de Pessoal de Nível Superior/Capes, responsável pelo acompanhamento dos cursos de pós-graduação em andamento no país, instituiu o Sistema de Avaliação da PósGraduação e realizou a primeira avaliação dos cursos de mestrado e doutorado existentes no Brasil, cujo foco foi aferir o nível de qualidade da pós-graduação brasileira. Desde então, os parâmetros e instrumentos utilizado têm sido objeto de aprimoramento contínuo (CAPES, 2017a).

\subsection{Avaliação da pós-graduação}

A consolidação da pós-graduação acelerou quando a CAPES, o CNPq e outros órgãos públicos ficaram incumbidos pelo decreto-lei № 464, de 11 de fevereiro de 1969, art. 36, de promover a "formação e o aperfeiçoamento do pessoal docente de ensino superior" e compor, para tanto, uma política nacional e regional definida pelo 
Conselho Federal de Educação e promovida por uma comissão executiva (Cury, 2005).

O Decreto 74.299 , de 18 de julho de 1974, alterou a estrutura da Capes, que passa a ser "órgão central superior, gozando de autonomia administrativa e financeira". A partir do novo Regimento Interno foi incentivada a colaboração com a direção do Departamento de Assuntos Universitários (DAU) na política nacional de pós-graduação, a promoção de atividades de capacitação de pessoal de nível superior, a gestão da aplicação dos recursos financeiros, orçamentários e de outras fontes nacionais e estrangeiras, bem como a análise e compatibilidade das normas e critérios do Conselho Nacional de Pós-Graduação (CAPES, 2017b).

Em 1981, por meio do Decreto № 86.791, a Capes é reconhecida como órgão responsável pela elaboração do Plano Nacional de Pós-Graduação stricto sensu, sendo também reconhecida como Agência Executiva do Ministério da Educação e Cultura junto ao Sistema Nacional de Ciência e Tecnologia, cabendo-Ihe elaborar, avaliar, acompanhar e coordenar as atividades relativas ao ensino superior, contribuindo, desta forma, com a criação de mecanismos efetivos de controle de qualidade dos cursos, estreitando, desse modo, sua relação com a comunidade científica e acadêmica (CAPES, 2017a).

O final da década de 1990 é considerado um marco importante na evolução do Programa de Acompanhamento e Avaliação da pós-graduação em virtude da sua informatização e da adoção de critérios mais rígidos para atribuição de notas aos programas. Desde então, "o sistema vem mantendo-se estável, com avanços incrementais em seus quesitos e itens de avaliação" (Maccari, Almeida, Riccio, \& Alejandro, 2014).

A avaliação dos programas de pós-graduação stricto sensu é feita com base em critérios comuns, ou seja, aplicáveis a todos os programas (acadêmicos ou não); por esta razão, é alvo de críticas, pois são tantas as variáveis em jogo que sempre se pode discordar do peso atribuído a cada uma delas. Nesta linha, nenhuma avaliação, por mais completa e rigorosa que seja, consegue captar toda a realidade, com seus múltiplos aspectos (InfoCapes, 2000).

Até o ano de 2013, a avaliação dos programas era trienal, com acompanhamento anual dos cursos pelas respectivas áreas. A partir de então, a 
avaliação passou a ser quadrienal, também com acompanhamento anual dos cursos pelas respectivas áreas (CAPES, 2014e)

Cabe destacar que o sistema de avaliação da pós-graduação brasileira é semelhante para todas as áreas do conhecimento, sendo que aproximadamente $80 \%$ dos quesitos são quantitativos e os $20 \%$ restantes de caráter qualitativo, mas que também podem ser quantificados, são eles: proposta do programa; corpo docente; corpo discente; produção intelectual; e inserção social. Os comitês de avaliação têm autonomia para modificar o peso dos quesitos em $5 \%$ para mais ou para menos (Maccari, 2008).

O mesmo autor afirma ainda que a avaliação do quesito "Proposta do Programa" é qualitativa, sendo classificada como "Adequada" ou "Inadequada". Nele são descritos o desenvolvimento do Programa ao longo dos anos; seu planejamento e perspectivas futuras; as inovações nas atividades de ensino, pesquisa e extensão; e como estão sendo realizadas as atividades de solidariedade e nucleação de outros programas.

Maccari (2008) avalia que o quesito "Corpo Docente" representa $20 \%$ do total da avaliação feita por meio de uma análise quantitativa, uma vez que os demais quesitos se subordinam a seu desempenho, revelando sua importância no sistema de avaliação. Os quesitos "Corpo Discente e Produção Intelectual" representam, cada um, 35\% da avaliação, estando suas análises quantitativas diretamente ligadas ao desempenho do quesito Corpo docente.

É no quesito "Inserção Social", equivalente a $10 \%$ da avaliação global, que a Comissão de Avaliação verifica a atuação dos Programas quanto a contribuição para a formação de docentes, em especial, para as regiões Norte e Nordeste. Também é relatada nesse quesito a participação do programa em projetos de cooperação com outros programas (Martins, 2013). Sua avaliação é uma das principais condições para que os programas alcancem as notas 5, 6 e 7 no ranking da CAPES (Maccari, 2008).

Visando aumentar a capilaridade dos Programas de Mestrado e Doutorado no Brasil, a CAPES fomenta, por meio de Editais, projetos Interinstitucionais, Minter e Dinter Nacionais e Internacionais. Essa estratégia de formação qualificada de recursos humanos contribui para o desenvolvimento sócio-econômico-cultural, científico-tecnológico, de inovação e possibilitando paralelamente a oferta de 
docentes para nucleação de novos programas de pós-graduação stricto sensu fora dos centros consolidados de ensino e pesquisa.

O sistema de avaliação da CAPES vem contribuindo para melhorar a qualidade dos programas de pós-graduação em Administração no Brasil. Assim, na área de Administração Pública e de Empresas, Ciências Contábeis e Turismo, os Programas têm utilizado os parâmetros, os dados e os resultados das avaliações como ponto de partida para definição de estratégias, de metas e de planos de ação, possibilitando, dessa maneira, um maior alinhamento às exigências desse Sistema e melhor direcionamento dos esforços e dos investimentos (Maccari, 2008).

\section{Metodologia}

Quanto à natureza da pesquisa, esta pode ser classificada como qualitativa, pois leva em consideração a existência de uma relação dinâmica entre o mundo real e o sujeito, isto é, um vínculo indissociável entre o mundo objetivo e a subjetividade do sujeito que não pode ser traduzido em números. O ambiente natural é a fonte direta para coleta de dados e o pesquisador é o instrumento chave (Clegg \& Hardy, 1999), e o rigor científico assegura a legitimidade dos dados gerados em sua utilização (Flick, 2008).

A pesquisa, em relação à abordagem metodológica, classifica-se como exploratória, uma vez que viabiliza estudos com dados qualitativos, particularmente úteis quando se quer entender o porquê do relacionamento entre variáveis (Eisenhardt, 1989). Dessa forma, ponderou-se que esse tipo de pesquisa possui o potencial para atender, de maneira satisfatória, aos interesses dos pesquisadores em se obter informações relevantes, resultantes do levantamento em profundidade dos dados, possibilitando conhecer melhor determinado fenômeno (Yin, 2015).

\subsection{Dados da pesquisa}

Os dados para a pesquisa foram coletados na Plataforma Sucupira/CAPES, por meio do sistema de busca nela disponibilizado, selecionando-se os cursos em funcionamento com notas 5, 6 e 7 da área de conhecimento de Administração Pública e de Empresas, Ciências Contábeis e Turismo. Como resultado, obteve-se uma lista 
com 27 cursos, sendo dois nota 7, três nota 6 e 22 nota 5 . Dos cursos nota 5, foram excluídos da amostra três cursos de pós-graduação em Ciências Contábeis (um da Fundação Instituto Capixaba de Pesquisa em Contabilidade Economia e Finanças/FUCAPE; um da Universidade Federal do Rio de Janeiro/UFRJ e um da Universidade do Vale do Rio dos Sinos/Unisinos) e um curso de pós-graduação em Contabilidade da Universidade de Brasília/UNB. Dos cursos nota seis, foi retirado da amostra o curso de Controladoria e Contabilidade da Universidade de São Paulo/USP. Além destes, também foram excluídos da pesquisa os cursos de mestrado profissional em Administração da Fundação Getúlio Vargas/FGV-RJ e da Pontifícia Universidade Católica de Minas Gerais/PUC/MG. O critério de exclusão se deve ao fato da avaliação destes cursos ser diferenciada. Estes ajustes foram necessários, pois, apesar de pertencer à área de Administração, estes cursos não abrangem esta área especificamente. Assim, para efeito de estudo, neste trabalho, considerar-se-ão 20 cursos, conforme Tabela 1:

\section{Tabela 1}

Programas em Administração nota 5,6 e 7

\begin{tabular}{ll|c|c|c}
\hline & PROGRAMA & INSTITUIÇÃo DE ENSINO & M & D \\
\hline $\begin{array}{l}\text { 1. } \\
\text { Emprinistração de }\end{array}$ & Fundação Getúlio Vargas/SP (FGV) - SP & 7 & 7 \\
\hline 2. & Administração & Universidade de São Paulo (USP) - SP & 7 & 7 \\
\hline 3. & Administração & Universidade Federal de Minas Gerais - (UFMG) - MG & 6 & 6 \\
\hline 4. & Administração & Fundação Getúlio Vargas/RJ (FGV) - RJ & 6 & 6 \\
\hline 5. & Administração & Universidade do Vale do Itajaí (UNIVALI) - SC & 5 & 5 \\
\hline 6. & Administração & Universidade Federal do Rio Grande do Norte (UFRN) - \\
RN & 5 & 5 \\
\hline 7. & Administração & Universidade Municipal de São Caetano do Sul (USCS) - \\
SP & 5 & 5 \\
\hline 8. & Administração & Universidade do Vale do Rio dos Sinos (UNISINOS) - RS & 5 & 5 \\
\hline 9. & Administração & Universidade Federal do Rio Grande do Sul (UFRGS) - \\
RS & 5 & 5 \\
\hline
\end{tabular}




\begin{tabular}{l|c|c|c}
\hline 10. Administração & Universidade Nove de Julho (UNINOVE) - SP & 5 & 5 \\
\hline $\begin{array}{l}\text { 11. Administração } \\
\begin{array}{l}\text { 12. Administração de } \\
\text { Empresas }\end{array}\end{array}$ & Universidade Federal de Lavras (UFLA) - MG & 5 & 5 \\
\hline $\begin{array}{l}\text { 13. Administração e } \\
\text { Negócios }\end{array}$ & Pniversidade Presbiteriana Mackenzie (UPM) - SP & 5 & 5 \\
\hline $\begin{array}{l}\text { 14. Administração } \\
\text { Pública e Governo }\end{array}$ & Fundação Getúlio Vargas/SP (FGV) - SP & 5 & 5 \\
\hline $\begin{array}{l}\text { 15. Administração } \\
\text { Pública }\end{array}$ & Fundação João Pinheiro - Escola de Governo (FJP) - MG & 5 & - \\
\hline $\begin{array}{l}\text { 16. Administração } \\
\text { 17. Administração de } \\
\text { Empresas }\end{array}$ & Pontifícia Universidade Católica do Paraná (PUC) - PR & 5 & 5 \\
\hline $\begin{array}{l}\text { 18. Administração } \\
\text { 19. Administração }\end{array}$ & Pontifícia Universidade Católica do Rio de Janeiro (PUC) & 5 & 5 \\
\hline 20. Administração & Universidade de Brasília (UNB) - DF & 5 & 5 \\
\hline
\end{tabular}

Fonte: Plataforma Sucupira/CAPES - dados extraídos em 11/07/2017

$\mathrm{D}=$ Doutorado, $\mathrm{M}=$ Mestrado

Dando sequência à coleta de dados, foram selecionados os programas que possuíam registro de oferta de Minter (mestrado interinstitucional) ou Dinter (doutorado interinstitucional) desde 2010, período válido para a avaliação de 2013 e 2017. Os dados coletados foram organizados por região receptora e relacionados na Tabela 2.

\section{Tabela 2}

Programas com registro de oferta de Dinter (D) e Minter (M)

\begin{tabular}{|c|c|c|c|c|}
\hline Tipo & Programa & $\begin{array}{l}\text { Instituição } \\
\text { Promotora }\end{array}$ & Instituição Receptora & $\begin{array}{l}\text { Período de } \\
\text { Vigência }\end{array}$ \\
\hline \multicolumn{5}{|c|}{ REGIÃO SUDESTE } \\
\hline DINTER & $\begin{array}{l}\text { Administração de } \\
\text { Empresas }\end{array}$ & $\begin{array}{l}\text { Fundação Getúlio } \\
\text { Vargas/SP }\end{array}$ & $\begin{array}{l}\text { Universidade Federal de } \\
\text { Ouro Preto (Ufop) - MG }\end{array}$ & $\begin{array}{l}02 / 02 / 2015 \\
\mathrm{a} \\
30 / 04 / 2019\end{array}$ \\
\hline DINTER & $\begin{array}{l}\text { Administração De } \\
\text { Empresas }\end{array}$ & $\begin{array}{l}\text { Pontifícia } \\
\text { Universidade } \\
\text { Católica do Rio de } \\
\text { Janeiro }\end{array}$ & $\begin{array}{l}\text { Instituto Federal de } \\
\text { Educacao, Ciencia e } \\
\text { Tecnologia do Sudeste de } \\
\text { Minas Gerais } \\
\text { (IFSUDESTEMG) - MG }\end{array}$ & $\begin{array}{l}02 / 01 / 2012 \\
\mathrm{a} \\
30 / 12 / 2016\end{array}$ \\
\hline DINTER & Administração & $\begin{array}{l}\text { Universidade } \\
\text { Federal de Lavras - } \\
\text { Mg }\end{array}$ & $\begin{array}{l}\text { Universidade Federal dos } \\
\text { Vales do Jequitinhonha e } \\
\text { Mucuri (UFVJM) - MG }\end{array}$ & $\begin{array}{l}02 / 02 / 2015 \\
\mathrm{a} \\
30 / 04 / 2019\end{array}$ \\
\hline \multicolumn{5}{|c|}{ Região Sul } \\
\hline
\end{tabular}




\begin{tabular}{|c|c|c|c|c|}
\hline DINTER & Administração & $\begin{array}{l}\text { Universidade } \\
\text { Municipal de São } \\
\text { Caetano do Sul }\end{array}$ & $\begin{array}{l}\text { Faculdade Meridional } \\
\text { (IMED) - RS }\end{array}$ & $\begin{array}{l}02 / 02 / 2015 \\
\text { a } \\
30 / 04 / 2019\end{array}$ \\
\hline DINTER & $\begin{array}{l}\text { Administração Pública } \\
\text { e Governo }\end{array}$ & $\begin{array}{l}\text { Fundação Getúlio } \\
\text { Vargas/SP }\end{array}$ & $\begin{array}{l}\text { Universidade Estadual de } \\
\text { Maringá (UEM) - PR }\end{array}$ & $\begin{array}{l}05 / 10 / 2012 \\
\mathrm{a} \\
31 / 12 / 2016 \\
\end{array}$ \\
\hline DINTER & Administração & $\begin{array}{l}\text { Universidade } \\
\text { Federal do Rio } \\
\text { Grande do Sul }\end{array}$ & $\begin{array}{l}\text { Universidade Comunitária } \\
\text { da Região de Chapecó } \\
\text { (UNOCHAPECÓ) - SC }\end{array}$ & $\begin{array}{l}05 / 10 / 2012 \\
\mathrm{a} \\
31 / 12 / 2016 \\
\end{array}$ \\
\hline MINTER & Administração & $\begin{array}{l}\text { Universidade Nove } \\
\text { de Julho }\end{array}$ & $\begin{array}{l}\text { Instituto De } \\
\text { Desenvolvimento } \\
\text { Educacional do Alto } \\
\text { Uruguai (IDEAU) - RS }\end{array}$ & $\begin{array}{l}05 / 02 / 2015 \\
a \\
05 / 04 / 2017\end{array}$ \\
\hline MINTER & $\begin{array}{l}\text { Administração de } \\
\text { Empresas }\end{array}$ & $\begin{array}{l}\text { Universidade } \\
\text { Presbiteriana } \\
\text { Mackenzie }\end{array}$ & $\begin{array}{l}\text { Centro Universitário } \\
\text { Dinâmica das Cataratas } \\
\text { (UDC) - PR }\end{array}$ & $\begin{array}{l}03 / 08 / 2015 \\
a \\
31 / 10 / 2017\end{array}$ \\
\hline MINTER & Administração & $\begin{array}{l}\text { Pontifícia } \\
\text { Universidade } \\
\text { Católica do Paraná }\end{array}$ & $\begin{array}{l}\text { Faculdade de Ciências } \\
\text { Sociais Aplicadas de } \\
\text { Cascavel (FCSAC) - PR }\end{array}$ & $\begin{array}{l}05 / 10 / 2012 \\
\mathrm{a} \\
31 / 12 / 2014\end{array}$ \\
\hline MINTER & $\begin{array}{l}\text { Administração e } \\
\text { Negócios }\end{array}$ & $\begin{array}{l}\text { Pontifícia } \\
\text { Universidade } \\
\text { Católica do Rio } \\
\text { Grande do Sul } \\
\end{array}$ & $\begin{array}{l}\text { Faculdade Assis Gurgacz } \\
\text { (FAG) - PR }\end{array}$ & $\begin{array}{l}05 / 10 / 2012 \\
a \\
31 / 12 / 2014\end{array}$ \\
\hline MINTER & Administração & $\begin{array}{l}\text { Universidade do } \\
\text { Vale do Rio dos } \\
\text { Sinos }\end{array}$ & $\begin{array}{l}\text { Centro Universitário Para } \\
\text { O Desenvol. Do Alto Vale } \\
\text { Do Itajaí (UNIDAVI) - SC }\end{array}$ & $\begin{array}{l}03 / 08 / 2016 \\
a \\
31 / 10 / 2018\end{array}$ \\
\hline \multicolumn{5}{|c|}{ Região Centro-Oeste } \\
\hline DINTER & $\begin{array}{l}\text { Administração de } \\
\text { Empresas }\end{array}$ & $\begin{array}{l}\text { Universidade } \\
\text { Presbiteriana } \\
\text { Mackenzie } \\
\end{array}$ & $\begin{array}{l}\text { Faculdade de Ciências } \\
\text { Sociais Aplicadas } \\
\text { (FACISA) - MT } \\
\end{array}$ & $\begin{array}{l}05 / 10 / 2012 \\
\mathrm{a} \\
31 / 12 / 2016 \\
\end{array}$ \\
\hline DINTER & Administração & $\begin{array}{l}\text { Universidade do } \\
\text { Vale Do Rio Dos } \\
\text { Sinos }\end{array}$ & $\begin{array}{l}\text { Universidade do Estado } \\
\text { de Mato Grosso } \\
\text { (UNEMAT) - MT }\end{array}$ & $\begin{array}{l}05 / 10 / 2012 \\
\mathrm{a} \\
31 / 12 / 2016 \\
\end{array}$ \\
\hline DINTER & Administração & $\begin{array}{l}\text { Universidade de } \\
\text { Brasília - DF }\end{array}$ & $\begin{array}{l}\text { Universidade Federal de } \\
\text { Goiás (UFG) - GO }\end{array}$ & $\begin{array}{l}02 / 01 / 2012 \\
\mathrm{a} \\
30 / 12 / 2016\end{array}$ \\
\hline \multicolumn{5}{|c|}{ Região Nordeste } \\
\hline DINTER & Administração & $\begin{array}{l}\text { Pontifícia } \\
\text { Universidade } \\
\text { Católica do Paraná }\end{array}$ & $\begin{array}{l}\text { Universidade Federal } \\
\text { Rural do Semi-Árido } \\
\text { (UFERSA) - RN } \\
\end{array}$ & $\begin{array}{l}02 / 01 / 2012 \\
\mathrm{a} \\
30 / 12 / 2016 \\
\end{array}$ \\
\hline DINTER & Administração & $\begin{array}{l}\text { Universidade } \\
\text { Federal do Rio } \\
\text { Grande do Sul } \\
\end{array}$ & $\begin{array}{l}\text { Universidade Federal de } \\
\text { Alagoas (UFAL) - AL }\end{array}$ & $\begin{array}{l}02 / 01 / 2012 \\
\mathrm{a} \\
30 / 12 / 2016 \\
\end{array}$ \\
\hline DINTER & Administração & $\begin{array}{l}\text { Universidade } \\
\text { Federal da Bahia }\end{array}$ & $\begin{array}{l}\text { Universidade Federal do } \\
\text { Ceará (UFC) - CE }\end{array}$ & $\begin{array}{l}02 / 01 / 2012 \\
\mathrm{a} \\
30 / 12 / 2016\end{array}$ \\
\hline DINTER & Administração & $\begin{array}{l}\text { Universidade } \\
\text { Federal da Bahia }\end{array}$ & $\begin{array}{l}\text { Fundação Universidade } \\
\text { Federal de Sergipe } \\
\text { (FUFSE) - SE }\end{array}$ & $\begin{array}{l}02 / 02 / 2015 \\
\mathrm{a} \\
30 / 04 / 2019 \\
\end{array}$ \\
\hline DINTER & Administração & $\begin{array}{l}\text { Universidade } \\
\text { Federal do Rio } \\
\text { Grande do Norte }\end{array}$ & $\begin{array}{l}\text { Universidade de } \\
\text { Pernambuco (UFPE) - PE }\end{array}$ & $\begin{array}{l}02 / 02 / 2015 \\
\mathrm{a} \\
30 / 04 / 2019 \\
\end{array}$ \\
\hline
\end{tabular}

Universidade

Municipal de São Caetano do Sul

Fundação Getúlio Vargas/SP

Federal do Rio

Universidade Nove de Julho

Universidade

Presbiteriana

Pontifícia

Universidade

Pontifícia

Católica do Rio

Universidade do

Vale do Rio dos

O Desenvol. Do Alto Vale

Do Itajaí (UNIDAVI) - SC

Região Centro-Oeste 


\begin{tabular}{|c|c|c|c|c|}
\hline MINTER & Administração & $\begin{array}{l}\text { Universidade do } \\
\text { Vale do Itajaí }\end{array}$ & $\begin{array}{l}\text { Faculdade Luciano Feijão } \\
\text { (FLF) - CE }\end{array}$ & $\begin{array}{l}05 / 02 / 2015 \\
a \\
05 / 04 / 2017\end{array}$ \\
\hline \multicolumn{5}{|c|}{ Região Norte } \\
\hline DINTER & Administração & $\begin{array}{l}\text { Universidade } \\
\text { Federal de Minas } \\
\text { Gerais - MG }\end{array}$ & $\begin{array}{l}\text { Universidade Federal do } \\
\text { Amazonas (UFAM) - AM }\end{array}$ & $\begin{array}{l}02 / 02 / 2015 \\
\mathrm{a} \\
30 / 04 / 2019\end{array}$ \\
\hline DINTER & Administração & $\begin{array}{l}\text { Universidade Nove } \\
\text { de Julho }\end{array}$ & $\begin{array}{l}\text { Universidade Federal de } \\
\text { Mato Grosso (UFMT) - } \\
\text { MT }\end{array}$ & $\begin{array}{l}02 / 02 / 2015 \\
\mathrm{a} \\
30 / 04 / 2019 \\
\end{array}$ \\
\hline MINTER & Administração & $\begin{array}{l}\text { Universidade } \\
\text { Federal de Santa } \\
\text { Maria }\end{array}$ & $\begin{array}{l}\text { Universidade Federal do } \\
\text { Maranhão (UFMA) - MA }\end{array}$ & $\begin{array}{l}02 / 01 / 2012 \\
\mathrm{a} \\
26 / 08 / 2014 \\
\end{array}$ \\
\hline MINTER & Administração & $\begin{array}{l}\text { Universidade } \\
\text { Federal do Rio } \\
\text { Grande do Sul } \\
\end{array}$ & $\begin{array}{l}\text { Universidade Federal do } \\
\text { Acre (UFAC) - AC }\end{array}$ & $\begin{array}{l}02 / 02 / 2015 \\
\mathrm{a} \\
30 / 04 / 2017 \\
\end{array}$ \\
\hline
\end{tabular}

Fonte: Plataforma Sucupira/CAPES - dados extraídos em 11/07/2017

Foram consultadas, ainda, na mesma Plataforma, as disciplinas ativas informadas pelos programas até o ano de 2016, com o objetivo de identificar registros de iniciativas de internacionalização na grade curricular dos programas pesquisados. Também foram analisados os regimentos de cada programa, buscando informações sobre ações de internacionalização.

Entendendo como ocorreu a coleta de dados, na próxima seção apresentaremos os resultados obtidos, fazendo uma discussão amparada no referencial teórico apresentado.

\section{Resultados e Discussão}

Em 2016, a área de avaliação Administração Pública e de Empresas, Ciências Contábeis e Turismo era composta por 184 programas; destes, 62 doutorados, 107 mestrados acadêmicos e 75 mestrados profissionais.

$\mathrm{Na}$ área básica de Administração foram encontrados 128 programas, sendo 69 acadêmicos e 59 profissionais. Dispostos na Figura 1, os dados utilizados nesta pesquisa revelam uma concentração dos programas de pós-graduação em Administração com melhores conceitos nas regiões sudeste e sul, deixando evidente as assimetrias regionais existentes na área. Esta situação é reconhecida pela coordenação da área de Administração Pública e de Empresas, Ciências Contábeis e Turismo da CAPES, que aponta como principal motivo de reprovação de cursos novos para a região norte, o baixo número de doutores na área. A coordenação afirma ainda que a fixação de doutores, com o respectivo aumento nas contratações destes 
profissionais, está equilibrando este problema na região centro-oeste (CAPES, 2017c).

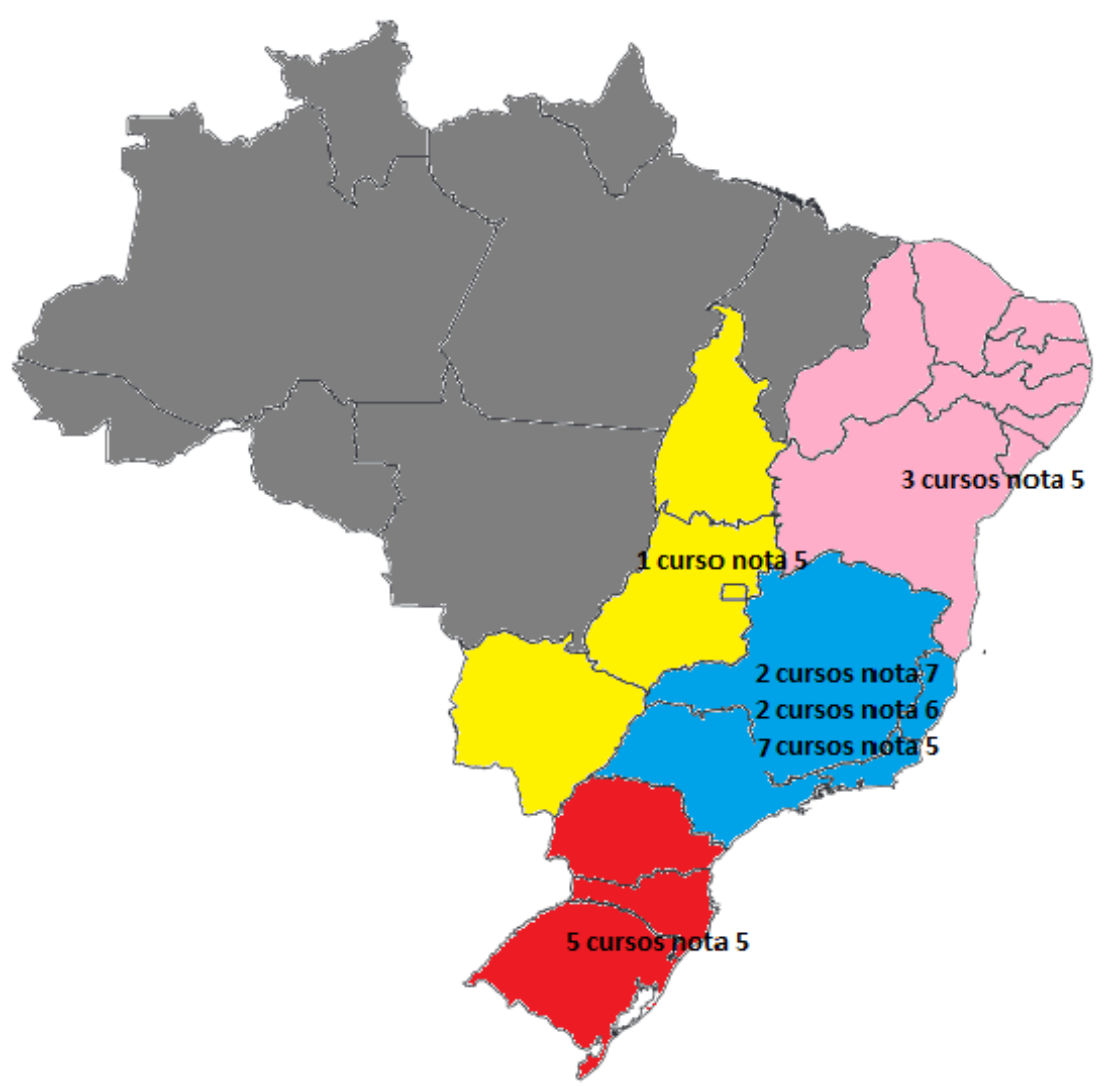

\section{Figura 1}

Distribuição regional dos cursos da área de administração Fonte: Plataforma Sucupira - dados extraídos em 18/01/2017

Em busca da consolidação ou de um melhor conceito por meio de avaliação realizada pela área e pelo Comitê Técnico Científico/CTC da CAPES, os programas procuram cumprir indicadores que resultam no conceito final atribuído a eles no período da avaliação. Na avaliação trienal de 2013, os indicadores utilizados foram: proposta do curso, corpo docente, corpo discente, teses e dissertações/trabalhos de conclusão, produção intelectual e inserção social.

Segundo a CAPES (2017d), a "estrutura curricular deve apresentar um núcleo de disciplinas ou seminários de tratamento metodológico relacionado à(s) área(s) de concentração além de um de disciplinas ou seminários com conteúdos relacionados às linhas de pesquisa". Como resultado da pesquisa realizada acerca das disciplinas obrigatórias ofertadas pelos PPG's em Administração com notas 5-6 e 7, identifica-se 
a preocupação com a coerência destas disciplinas com as áreas de concentração de cada programa. No entanto, apresentou-se uma tendência de oferta de disciplina optativas em substituição às disciplinas obrigatórias para cumprimento de créditos regimentais para conclusão do curso. Nestes casos, a grade curricular dos cursos também mantinha relação direta com a área de concentração, apesar de estar, muitas vezes, distribuídas em linhas de pesquisa. Essa tendência reflete a intenção dos programas em procurar ajustar sua estrutura acadêmica com seu objetivo final de formar profissionais altamente qualificados, que possam contribuir para o desenvolvimento científico, tecnológico e social do país, conforme preconiza o Plano Nacional de Pós-graduação vigente.

Outra tendência verificada é a oferta de disciplinas em língua estrangeira. Dos 20 programas pesquisados, oito ofertam disciplinas em inglês, sendo que, destes, destaca-se o programa da Fundação Getúlio Vargas/SP, inovando ao ofertar integralmente seu curso em inglês, além do incentivo, por todos os programas pesquisados, para a realização do estágio sanduíche em instituições estrangeiras parceiras, o que permite "maior possibilidade de inovação" na formação do aluno. Com estas iniciativas, os programas direcionam suas ações para cumprir com um dos objetivos previstos na ficha de avaliação da área, que prevê a indicação de "planejamento do programa com vistas a seu desenvolvimento futuro, contemplando os desafios internacionais da área na produção do conhecimento, seus propósitos na melhor formação de seus alunos, suas metas quanto à inserção social mais rica dos seus egressos, seguindo os parâmetros da área". Marrara (2007) afirma que a internacionalização na pós-graduação é um processo de cooperação internacional exigido para que um programa de pós-graduação amplie a capacitação de seus discentes e docentes, estimulando o crescimento da pesquisa e a solução de problemas regionais, sem deixar de lado os objetivos meramente institucionais.

Neste sentido, um programa, dentre os que possuem conceito 5, que obteve destaque foi o da Universidade Nove de Julho/Uninove, que, além de disciplinas ofertadas em língua inglesa, faz constar em sua grade curricular, de forma obrigatória para o cumprimento dos créditos regimentais, a realização de módulo internacional, que deve ser realizado em uma universidade de excelência no exterior. Observou-se com os dados que, mesmo entre os cursos com conceitos 6 e 7 , onde existe a obrigatoriedade da internacionalização, com exceção do programa da Fundação 
Getúlio Vargas/FGV-SP, não consta em seus regimentos a exigência de cumprimento de créditos obrigatórios em cursos realizados no exterior. Nestes cursos, os discentes são fortemente incentivados a realizar um estágio sanduíche no exterior, procurando cumprir de outras formas o requisito de internacionalização exigido na avaliação/CAPES. Entre os programas nota 5, à exceção do programa da Universidade Nove de Julho/Uninove, as ações de internacionalização se restringem à oferta de algumas disciplinas em inglês e ao incentivo para realização do estágio sanduíche no exterior.

De acordo com Stallivieri (2004, p. 31) o diferencial na qualidade de um programa pode ser verificado pelo " nível de excelência das parcerias e na qualidade das oportunidades que as universidades têm a oferecer aos seus estudantes e professores de adquirirem, durante sua vida acadêmica, uma experiência no exterior" e a internacionalização vista como "um passo necessário para encorajar o desenvolvimento equilibrado das nações através da educação" (Stallivieri, 2017, p. 19).

Para atender parcialmente a outro importante indicador da ficha de avaliação da CAPES, o de inserção social, os programas de pós-graduação em administração ofertam cursos de mestrado e doutorado interinstitucionais, chamados de Minter e Dinter, respectivamente. Entre as duas modalidades, destaca-se a oferta de Dinter, o que reforça a colaboração dos programas com a qualificação de recursos humanos e de nucleação de grupos de pesquisa em outros estados e regiões do país, atendendo a critérios de avaliação da CAPES, em especial para os cursos 6 e 7 . 


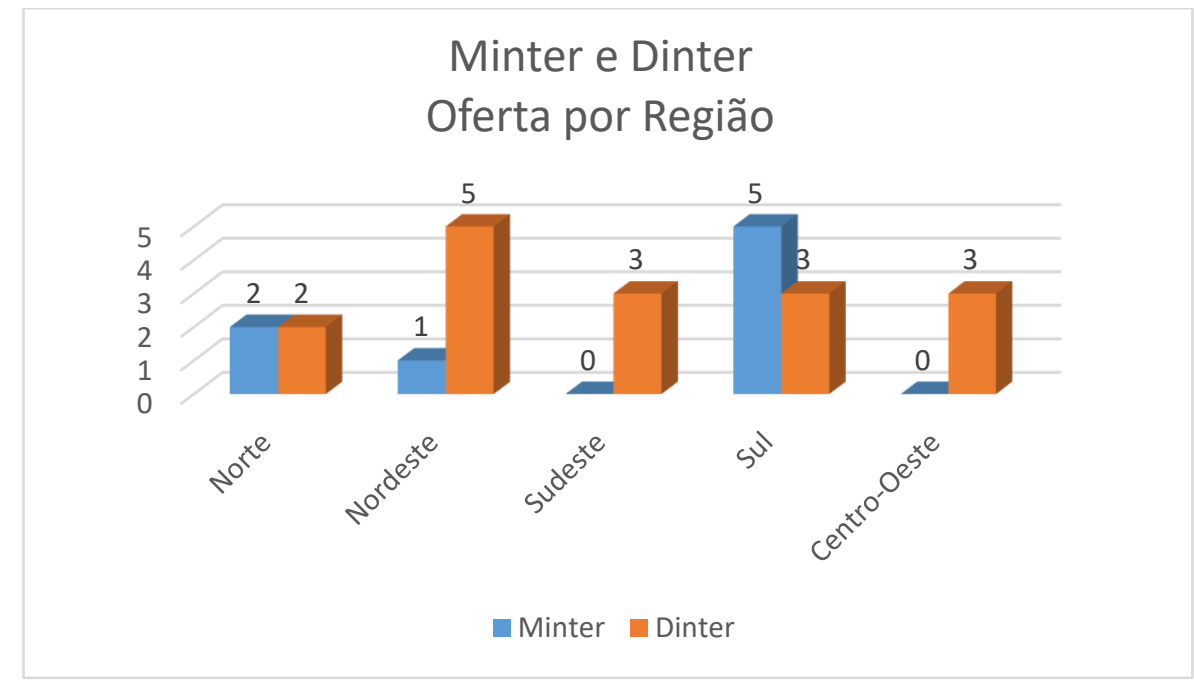

\section{Figura 2}

Oferta de Minter e Dinter por região

Fonte: Plataforma Sucupira/CAPES

Os dados revelam, ainda, uma concentração de oferta de programas interinstitucionais para a região sul, apesar desta região possuir um número considerável de programas de pós-graduação em administração recomendados pela CAPES. Por outro lado, identificou-se que as regiões norte e centro-oeste, embora sejam regiões menos favorecidas em número de cursos na área e historicamente problemáticas na qualificação e retenção de professores doutores, as ofertas de Minter e Dinter foram modestas. Uma justificativa para este fato pode estar no financiamento do projeto, por vezes mais elevado para estas regiões ou as incertezas em assumir compromissos estabelecidos no edital como, por exemplo, "garantir no desenvolvimento dos Projetos de Minter e de Dinter o mesmo nível de qualidade que caracteriza o curso oferecido em sua sede, submetendo a iniciativa aos mesmos controles e exigências acadêmicas" para a instituição promotora ou "dispor dos recursos de infraestrutura de ensino e pesquisa considerados indispensáveis para o projeto" para a instituição receptora.

Os resultados demonstram que a procura pela excelência na avaliação da CAPES levam os programas a se tornarem parecidos em sua essência, o que encontra amparo na literatura quando DiMaggio e Powell (1983) afirmam que, a partir do momento que se consolidam, as organizações tendem a se tornarem cada vez mais homogêneas, ou seja, passam pelo processo de isomorfismo que, neste caso, enquadra-se no isomorfismo coercitivo, representando o resultado tanto de pressões formais quanto de pressões informais exercidas sobre as organizações por outras 
organizações das quais elas dependem. Isso parece ser aplicável à relação entre a pós-graduação e a CAPES e seu processo avaliativo, já que instituições que têm seus programas consolidados e planejamento estratégico de aprimoramento tendem a ser melhor avaliadas que outras em fase inicial.

Nesse ambiente isomórfico, os dados evidenciam que os programas buscam inovar atuando de forma incremental, incluindo em suas estruturas curriculares não só disciplinas em outro idioma, mas também ofertando disciplinas que trabalham na fronteira do conhecimento, nelas incluindo as faces da criatividade e inovação. Além disso, entre as instituições privadas, pode ser mencionado uma inovação incremental no âmbito social, o "programa de bolsas taxa" implementados por três instituições privadas, com o objetivo de fortalecer a pesquisa e a integração graduação-pósgraduação, com a atuação destes bolsistas como tutores naquele nível de ensino, contribuindo para uma melhor formação do Bacharel em Administração.

A análise da expansão no âmbito social por meio da internacionalização pela CAPES dos Programas corrobora o previsto no Plano Nacional de Pós-graduação 2011-2020 (CAPES, 2010); neste quesito, duas instituições da amostra se destacam: a Fundação Getúlio Vargas/FGV-SP e a Universidade Nove de Julho/Uninove. A primeira, pelo pioneirismo em ofertar um programa totalmente em língua estrangeira. A segunda, por incluir como obrigatória a realização de um módulo de disciplina no exterior, deslocando a turma inteira para a realização uma experiência de alto impacto acadêmico, proporcionando aos discentes vivenciar a dinâmica da criatividade e da inovação na perspectiva de um ambiente sociocultural específico e propício, além de identificar e caracterizar elementos que facilitam e dificultam essa dinâmica, tendo como objetivo desenvolver em seus discentes a competência de "atuar criativamente".

De acordo com Stallivieri (2017, p. 20), as vantagens da internacionalização das instituições de ensino superior são a expansão de sua projeção intelectual, uma participação mais ativa em fóruns globais, o destaque dos pesquisadores no cenário internacional, fomentando parcerias com profissionais estrangeiros ou sua "participação em redes de conhecimento universal, podendo assim identificar problemas comuns, encontrar soluções para esses problemas e também estabelecer fortes laços de comunicação fluida constante" contribuindo para o desenvolvimento regional onde a instituição está localizada. 


\section{Considerações finais}

Apesar de todas as políticas implementadas nos últimos anos, como resultado do estudo observou-se ainda uma assimetria regional na distribuição dos programas de pós-graduação em Administração com melhores conceitos, situação reconhecida pela coordenação de área na CAPES, que busca caminhos para sua solução.

Neste trabalho procurou-se verificar como poderia ocorrer a inovação nos Programas de Pós-graduação em Administração stricto sensu em um ambiente isomórfico e se, apesar do ambiente isomórfico, imposto pelo sistema de avaliação a todos, existem aqueles que conseguem se destacar por meio de estratégias diferenciadas, que contribuem para a qualidade percebida no processo avaliativo.

Os resultados mostram que, na busca pela excelência no processo avaliativo da CAPES, os programas passam por processo de isomorfismo coercitivo, pois, pressionados pelos mesmos requisitos, tendem a se tornar cada vez mais homogêneos, o que pode ser observado na semelhança da grade curricular ofertada pelos programas, bem como nas ações utilizadas para alcançar metas do quesito "inserção social", especialmente com oferta de MINTER e DINTER, programas que deveriam ser ofertados para regiões mais carentes de recursos humanos qualificados. No entanto, ficou evidenciado que sua ocorrência concentra-se em regiões privilegiadas, as quais já possuem cursos de excelência na área de Administração, o que aponta para necessidade de aprofundar a reflexão sobre esse cenário.

Apesar do ambiente isomórfico, em que é esperada uma homogeneidade, foi possível identificar ações inovadoras referentes aos quesitos de avaliação que abrangem a inserção social e a internacionalização. Como destaque, citam-se o programa de bolsas taxa instituídos pela Fundação Getúlio Vargas/SP, Fundação Getúlio Vargas/RJ e pela Universidade Nove de Julho/SP e a oferta do módulo internacional obrigatório, incluído na grade curricular do programa de pós-graduação em Administração da Universidade Nove de Julho/SP.

A utilização de programas da área básica de administração, com conceitos 5-6 e 7 são fatores limitantes da pesquisa, além da mesma se ater apenas a fontes documentais, sem utilização de entrevistas ou questionários com as instituições envolvidas para que uma análise circunstanciada fosse realizada. Esta seria uma 
sugestão para trabalhos futuros, nos quais o assunto poderia ser ampliado e aprofundado, visto sua relevância para o desenvolvimento da Pós-graduação no Brasil e definição de Políticas para o melhor uso de recursos públicos.

\section{Referências}

Balbachevsky, E. (2005). A pós-graduação no Brasil: novos desafios para uma política bem sucedida. In C. Brock, \& S. Schwartzman. Os desafios da educação no Brasil. Rio de Janeiro: Nova Fronteira.

Barreto, M. O., \& Martínez, M. A. (2007). Possibilidades criativas de professores em cursos de pós-graduação stricto sensu. Estudos de Psicologia, 24(4), p.463-473 Recuperado de http://repositorio.unb.br/handle/10482/6263

Belloni, J. A. (2000, Abril). Uma metodologia de avaliação da eficiência produtiva de universidades federais brasileiras. (Tese de doutorado em Engenharia da Produção. Universidade Federal de Santa Catarina, SC, Brasil). Recuperado de https://repositorio.ufsc.br/bitstream/handle/123456789/78457/153160.pdf?seque nce $=1$

Bessant, J., Tidd, J., \& Pavitt, K. (2008). Gestão da inovação (3a ed.). Porto Alegre: Bookman.

Boava, D. L. T., Macedo, F. M. F., Magalhães, L. H., \& Silva, R. D. da. (2013). Utilização da técnica laddering em estudos sobre valor em inovação. Gestão \& Planejamento-G\&P, 14(2). Recuperado de http://www.revistas.unifacs.br/index.php/rgb/article/view/1260

Bomeny, H. (2001). Newton Sucupira e os rumos da educação superior. Paralelo 15, CAPES, 128.

Bruno-Faria, M. de F. (2003). Criatividade, inovação e mudança organizacional. In S. M. V. Lima (Org.). Mudança organizacional: teoria e gestão. (pp. 111-142). Rio de Janeiro: Editora FGV.

Coordenação de Aperfeiçoamento de Pessoal de Nível Superior - CAPES. (2010). Plano nacional de pós-graduação [PNPG] 2011-2020. Brasília: CAPES.

Recuperado em 26 maio, 2018, de https://www.capes.gov.br/images/stories/download/Livros-PNPG-Volume-IMont.pdf

Coordenação de Aperfeiçoamento de Pessoal de Nível Superior - CAPES. (2017d). Documento de Área e Comissão - Trienal. Recuperado em 5 fevereiro, 2017, de http://www.avaliacaotrienal2013.capes.gov.br/documento-de-area-e-comissao

Coordenação de Aperfeiçoamento de Pessoal de Nível Superior - CAPES. (2017b). Evolução do sistema de avaliação da pós-graduação. Recuperado em 4 fevereiro, 2017, de 
http://www.capes.gov.br/images/stories/download/avaliacao/avaliacaon/evolucao-da-avaliacao-enviado-ed-26fev.pdf

Coordenação de Aperfeiçoamento de Pessoal de Nível Superior - CAPES. (2017a). História e missão. Recuperado em 5 fevereiro, 2017, de http://www.capes.gov.br/historia-e-missao

Coordenação de Aperfeiçoamento de Pessoal de Nível Superior - CAPES. (2014). Resolução CAPES no 5, Pub. L. No. 5. Recuperado de http://capes.gov.br/images/stories/download/avaliacao/legislacao/1412015resolucao-n-5-de11122014.pdf

Christensen, C. M. (2003). The innovator's dilemma: The revolutionary book that will change the way you do business. New York: Harper Business Essentials New York. Recuperado de http://globalloveins.com/book4/19066.pdf

Clegg, S. R., \& Hardy, C. (Eds.). (1999). Studying organizations: Theory and Methods. London: Sage Publications.

Conselho Nacional de Desenvolvimento Científico e Tecnológico - CNPQ. (2017). Centro de Memória - CNPq. Recuperado em 5 fev., 2017, de http://centrodememoria.cnpq.br/Missao2.html

Cunha, A. G. da, \& Sobrinho, C. M. (2007). Dicionário etimológico da língua portuguesa. Rio de Janeiro: Lexikon Ed. Digital.

Cury, C. R. J. (2005). Quadragésimo ano do parecer CFE n. 977/65. Revista Brasileira de Educação, 30, 7-20.

Decreto $n^{\circ}$ 5563. (2005). Regulamenta a Lei no 10.973, de 2 de dezembro de 2004, que dispõe sobre incentivos à inovação e à pesquisa científica e tecnológica no ambiente produtivo, e dá outras providências. Recuperado em 4 fev., 2017, de http://www.planalto.gov.br/ccivil_03/_ato2004-2006/2005/Decreto/D5563.htm

DiMaggio, P. J., \& Powell, W. W. (1983). The Iron Cage Revisited: Institutional Isomorphism and Collective Rationality in Organizational Fields. American Sociological Review, 48(2), 147-160. https://doi.org/10.2307/2095101

Dowling, J., \& Pfeffer, J. (1975). Organizational Legitimacy: Social Values and Organizational Behavior. The Pacific Sociological Review, 18(1), 122-136. https://doi.org/10.2307/1388226

Eisenhardt, K. M. (1989). Building Theories from Case Study Research. Academy of Management Review, 14(4), 532-550. https://doi.org/10.5465/AMR.1989.4308385

Fennell, M. L. (1980). The effects of environmental characteristics on the structure of hospital clusters. Administrative science quarterly, 25(3), 485-510. 
Flick, U. (2008). Introdução à Pesquisa Qualitativa (3a ed.). Porto Alegre: Artmed Editora. Recuperado de https://books.google.com.br/books?hl=pt$B R \& \mid r=\& i d=d K m q D A A A Q B A J \& o i=f n d \& p g=P A 1 \& d q=U m a+i n t r o d u \% C 3 \% A 7 \% C 3$ $\% \mathrm{~A} 30+\% \mathrm{C} 3 \% \mathrm{~A} 0+$ pesquisa++qualitativa $+.+\& \mathrm{ots}=\mathrm{JflgPW} 5 \mathrm{Jth} \& \mathrm{sig}=\mathrm{gFY} 3-$ 8SV1aTtLnY7QVk0w-EpBt4

Gualberto, S. S., \& Moreira, M. F. (2013). Dos modelos teóricos à gestão empírica da inovação em empresas de serviços de software do Distrito Federal. Revista Psicologia Organizações e Trabalho, 13(3), 339-350. Recuperado em 26 de maio, 2018, de http://pepsic.bvsalud.org/scielo.php?script=sci_arttext\&pid=S1984$66572013000300010 \& \operatorname{lng}=p t \&$ tIng $=p$

Guarido Filho, E. R., \& Machado-da-Silva, C. L. (2001). A influência de valores ambientais e organizacionais sobre a aprendizagem organizacional na indústria alimentícia paranaense. Revista de Administração Contemporânea, 5(2), 33-63. https://doi.org/10.1590/S1415-65552001000200003

Hawley, A. H. (1968). Human Ecology//International Encyclopedia of Social Sciences. NY: Mancmillan, 328-337.

Hirsch, P. M. (1972). Processing Fads and Fashions: An Organization-Set Analysis of Cultural Industry Systems. American Journal of Sociology, 77(4), 639-659.

Hirsch, P. M. (1975). Organizational Effectiveness and the Institutional Environment. Administrative Science Quarterly, 20(3), 327-344. https://doi.org/10.2307/2391994

InfoCapes. (2000). InfoCapes- Boletim Informativo. InfoCapes- Boletim Informativo, 8(CAPES). Recuperado de http://www.capes.gov.br/publicacoes/publicacoesantigas

Maccari, E. A. (2008). Contribuições à gestão dos programas de pós-graduação stricto sensu em administração no Brasil com base nos sistemas de avaliação norte americano e brasileiro.(Tese de Doutorado em Administração. Universidade de São Paulo, São Paulo, SP, Brasil).

Maccari, E. A., Almeida, M. I. R. de, Riccio, E. L., \& Alejandro, T. B. (2014). Proposta de um modelo de gestão de programas de pós-graduação na área de Administração a partir dos sistemas de avaliação do Brasil (CAPES) e dos Estados Unidos (AACSB). Revista de Administração, 49(2), 280-290.

Machado-da-Silva, C. L. (2003). Respostas estratégicas da administração e contabilidade ao sistema de avaliação da CAPES. Organizações \& Sociedade, 10(28), 63-82.

Machado-da-Silva, C. L., Fonseca, V. S., \& Fernandes, B. H. R. (1999). Mudança e estratégia nas organizações: perspectivas cognitiva e institucional. In M.M. F. Vieira, \& L. M. B. de Oliveira. (Orgs.). Administração contemporânea: perspectivas estratégicas. (pp. 102-118). São Paulo: Atlas. 
March, J. G., \& Olsen, J. P. (1976). Organizational choice under ambiguity. Ambiguity and choice in organizations, 2, 10-23.

Marrara, T. (2007). Internacionalização da Pós-Graduação: objetivos, formas e avaliação. Revista Brasileira de Pós-Graduação, 4(8). Recuperado de http://ojs.rbpg.capes.gov.br/index.php/rbpg/article/view/132

Martínez, M. A. (1997). Cómo desarrollar la creatividad en la escuela. Curso prereunión. Pedagogía, 97.

Martins, C. B., Maccari, E. A., Storopoli, J. E., \& Andrade, R. O. B. de. (2013). Influência das estratégias e recursos para o desenvolvimento dos programas de pós-graduação da área de Administração, Ciências Contábeis e Turismo no período de 2001 a 2009. Revista Gestão Universitária na América Latina GUAL, 6(3), 146-168.

McNeil, K., \& Minihan, E. (1977, setembro). Regulation of Medical Devices and Organizational Behavior in Hospitals. Administrative Science Quarterly, 475-490.

Meyer, J. W. (1979). The Impact of the Centralization of Educational Funding and Control on State and Local Organizational Governance. Recuperado de http://eric.ed.gov/?id=ED202139

Meyer, J. W., \& Rowan, B. (1977). Institutionalized Organizations: Formal Structure as Myth and Ceremony. American Journal of Sociology, 83(2), 340-363.

Miles, I. (2005). Innovation in services. In J. Fagerberg, D. C. Mowery, \& R. Nelson (Eds). The Oxford handbook of innovation. (pp. 433-458). Oxford: Oxford University Press.

Moritz, G. de O., Pereira, M. F., Moritz, M. O., \& Maccari, E. A. (2013). A PósGraduação brasileira: evolução e principais desafios no ambiente de cenários prospectivos. Future Studies Research Journal: Trends and Strategies, 5(2), 0334.

Nelson, R., \& Winter, S. (2005). Uma teoria evolucionária da mudança econômica. Campinas: Editora Unicamp.

Paixão, D. de B., Barbosa, J. S., \& Neves, K. P. (2009). A formação do bibliotecário no Brasil: quem forma o profissional da informação na região Sudeste. Anais do $10^{\circ}$ Encontro Regional de Estudantes de Biblioteconomia, Documentação, Ciência da Informação e Gestão da Informação da região Sudeste e CentroOeste, Universidade Federal de Goiás, GO, Brasil. Recuperado de https://portais.ufg.br/up/75/o/a_formacao_do_bibliotec_rio_no_brasil.pdf

Palma, G., \& Forster, M. M. dos S. (2011). Inovação e Educação Superior-rupturas e continuidades. Educação Unisinos, 15(2), 149-157.

Ribeiro, P. D. (2016). Relação entre internacionalização e inovação nas empresas: um estudo de caso. Revista do BNDES, 46, 263-309. 
Santos, C. M. dos. (2003). Tradições e contradições da pós-graduação no Brasil. Educação \& Sociedade, 24(83), 627-641. https://doi.org/http://dx.doi.org/10.1590/S0101-73302003000200016

Schumpeter, J. A. (1982). Teoria do desenvolvimento econômico: uma investigação sobre lucros, capital, crédito, juro e o ciclo econômico. São Paulo: Abril Cultural,

Sucupira, N. (1980). Antecedentes e primórdios da pós-graduação. In Fórum educacional (Vol. 4, p. 3-18). Recuperado de

http://bibliotecadigital.fgv.br/ojs/index.php/fe/article/viewFile/60545/58792

Stallivieri, L. (2004) Estratégias de internacionalização das universidades brasileiras. Caxias do Sul: EDUCS.

Stallivieri, L. (2017). Compreendendo a internacionalização da educação superior. Revista de Educação do Cogeime, 26 (50). 15-36.

Yin, R. K. (2015). Estudo de Caso-: Planejamento e Métodos. Porto Alegre: Bookman. Recuperado de https://books.google.com/books?hl=en\&lr=\&id=EtOyBQAAQBAJ\&oi=fnd\&pg=PR $1 \& \mathrm{dq}=\mathrm{YIN},+\mathrm{R} .+\mathrm{K} .+$ Estudo+de+caso:+planejamento+e+m\%C3\%A9todos.+3.+ed. +Porto+Alegre:+Bookman,+2005\&ots=-jcgjoEZAB\&sig=0oGivamB3VYngEmK57_P2VnR1Y

Zald, M. N. (1978). On the social control of industries. Social Forces. A Scientific Medium of Social Study and Interpretation, 57(1), 79-102. 